\title{
Implication of surface modified NZVI particle retention in the porous media: Assessment with the help of 1-D transport model
}

\author{
Trishikhi Raychoudhury ${ }^{1, *}$ and Vikranth Kumar Surasani ${ }^{2}$ \\ ${ }^{1}$ Department of Civil and Environmental Engineering, Indian Institute of Technology Patna, Bihta, \\ Patna, Bihar 801 103, India. \\ ${ }^{2}$ Department of Chemical Engineering, Birla Institute of Technology and Science Pilani-Hyderabad Campus, \\ Hyderabad 500 078, India. \\ *Corresponding author. e-mail: trishikhi@iitp.ac.in
}

MS received 2 February 2016; revised 3 February 2017; accepted 3 February 2017; published online 15 June 2017

Retention of surface-modified nanoscale zero-valent iron (NZVI) particles in the porous media near the point of injection has been reported in the recent studies. Retention of excess particles in porous media can alter the media properties. The main objectives of this study are, therefore, to evaluate the effect of particle retention on the porous media properties and its implication on further NZVI particle transport under different flow conditions. To achieve the objectives, a one-dimensional transport model is developed by considering particle deposition, detachment, and straining mechanisms along with the effect of changes in porosity resulting from retention of NZVI particles. Two different flow conditions are considered for simulations. The first is a constant Darcy's flow rate condition, which assumes a change in porosity, causes a change in pore water velocity and the second, is a constant head condition, which assumes the change in porosity, influence the permeability and hydraulic conductivity (thus Darcy's flow rate). Overall a rapid decrease in porosity was observed as a result of high particle retention near the injection points resulting in a spatial distribution of deposition rate coefficient. In the case of constant head condition, the spatial distribution of Darcy's velocities is predicted due to variation in porosity and hydraulic conductivity. The simulation results are compared with the data reported from the field studies; which suggests straining is likely to happen in the real field condition.

Keywords. Nanoparticles; porosity; transport; straining; deposition; flow condition.

\section{Introduction}

Nanoscale Zero-Valent Iron (NZVI) particles have the potential to remediate a variety of different organic contaminants and heavy metals in the aqueous phase (Tratnyek and Johnson 2006; O’Carroll et al. 2012; Raychoudhury and Scheytt
2013). However, the main concern is the NZVI particles tend to aggregate and retain in the pore spaces, which limits their application for in situ groundwater remediation (Petosa et al. 2010). Several studies, from last decade, have focused on surface modification of NZVI particles by coating the metallic surface with different

Supplementary material pertaining to this article is available on the Journal of Earth System Science website (http://www. ias.ac.in/Journals/Journal_of_Earth_System_Science). 
polymers and polyelectrolytes. An electrosteric repulsion force is induced between two colloid particles due to surface modification. This induced repulsion improves the colloidal stability and transport efficiency of the particles in the porous media (Saleh et al. 2007; Cirtiu et al. 2011). Numerous laboratory experiments have investigated the influence of water chemistry such as $\mathrm{pH}$, ionic strength, etc. (Saleh et al. 2008; Fatisson et al. 2010; Kim et al. 2012); flow rates (He et al. 2009; Raychoudhury et al. 2010); characteristics of modified-NZVI particles such as size, particle concentrations, types of surface modifiers, effect of aggregation, etc. (Phenrat et al. 2009; Raychoudhury et al. 2010, 2012); and porous media properties such as effect of sand size, mineral composition, presence of organic matter, etc. (Phenrat et al. 2010a; Kim et al. 2012; Raychoudhury et al. 2014) on the transport behaviour of modified-NZVI particles. Laboratory studies have also explored the transport behaviour of modified NZVI in two-dimensional sand packed systems (Kanel et al. 2008; Phenrat et al. 2010b). In summary, transport efficiency of modified-NZVI particles increased significantly $(50 \%-98 \%$ particles are eluted) when compared to unmodified NZVI particles (He et al. 2009; Phenrat et al. 2009; Raychoudhury et al. 2014; Laumann et al. 2014).

Different factors, which are relevant for natural groundwater conditions, such as ionic strength (IS), NZVI particle size, sand grain size, flow rate, etc., can influence the retention of modifiedNZVI particles in the porous media by different mechanisms. For example, the increase in particle deposition with increasing IS is typically attributed to the increase in attachment efficiency resulting from charge screening (Saleh et al. 2008; Fatisson et al. 2010; Laumann et al. 2014). Few studies (He et al. 2009; Raychoudhury et al. 2010) have shown that transport efficiency of modified NZVI particles increases with an increase in flow rate. Hydrodynamic drag acting on the deposited particles are higher at a higher flow rate, resulting in higher particle detachment and better transport (Bergendahl and Grasso 2000). Furthermore, reduction in flow rate can increase the collision frequency between the particle and the sand grain, which eventually enhances deposition in porous media (He et al. 2009). Particle size and aggregation kinetics of modified-NZVI particles can also influence particle deposition by altering the single collector contact efficiency (collision frequency between the sand grain and colloid particle, $\eta_{0}$ ) as demonstrated in the literature (Raychoudhury et al. 2012, 2014). Phenrat et al. (2009) have observed that at high $(\mathrm{g} / \mathrm{l})$ concentrations, the formation of big aggregates of carboxymethyl cellulose (CMC) modified-NZVI particles resulted in more particle retention when compared to that of smaller sized particles in less $(\mathrm{mg} / \mathrm{l})$ concentrated suspension. Effect of sand size on CMC-NZVI transport and occurrence of straining, which is clogging of small pores near the column inlet, is reported in the literature (Hong et al. 2009; Tosco and Sethi 2010; Raychoudhury et al. 2014).

Several studies have suggested that even with surface modification, a significant amount of NZVI particles are retained (due to combined effect of several mechanisms) in the porous media within a very small distance from the point of injection (Bennett et al. 2010; He et al. 2010; Johnson et al. 2013; Su et al. 2013). However, based on authors knowledge, only a single research group has explored the possible effect of NZVI particle retention on the porous media properties (Tosco and Sethi 2010; Hosseini and Tosco 2013). In practice, NZVI particles are injected at a very high concentration in the field. Therefore, it is most likely that significant mass of NZVI would be retained in a localized area (Raychoudhury et al. 2014), which may alter porous media properties (Hosseini and Tosco 2013). Thus, it is extremely important to understand the possible effect of CMCNZVI particles retention on the porous media properties and its implication on further CMCNZVI transport. A previous study (Raychoudhury et al. 2014) has demonstrated the significance of straining and deposition with the help of column experiment and 1-D colloid transport model. However, the model has a limited scope that does not include the change in porous media properties (porosity, permeability, etc.) resulting from particle retention. Furthermore, in the natural system groundwater flows due to the difference in the hydraulic head under Darcy's velocity. Change in porosity and hydraulic conductivity will influence groundwater flow and transport of NZVI in the local scale, which was not included in the previous study. A mathematical model has enormous potential to forecast the possible out come with varying input conditions. Thus, the present study will not only help to understand the fate of CMC-NZVI particles in the porous media under different conditions but will also be useful to the research community in designing laboratory and field scale experiment for further study. 
The main objectives of this paper are, therefore, to understand the possible effect of CMC-NZVI particle retention on the porous media properties (i.e., porosity, hydraulic conductivity, etc.) and consequently its influence on further particle retention under different flow conditions, with the help of 1-D colloid transport model. In this study, we would like to assess the significance of different mechanisms such as deposition, straining, and detachment on the retention of CMC-NZVI particles through porous media having different porous media properties. A 1-D transport model was developed by considering attachment, detachment, and straining mechanisms along with the effect of a change in porosity resulting from retention of NZVI particles. Three scenarios were considered having different flow conditions and porous media properties. In the first scenario, porous media properties, and the experimental conditions are taken same as reported in Raychoudhury et al. (2014) and given in table 1 . The implications of CMC-NZVI retention on the porous media are evaluated. The value of estimated parameters from the model fit, with and without incorporating the change in porous media properties are compared. For the second scenario, two hypothetical porous media with different porous media properties and flow rates are considered, and the significance of different mechanisms on particle transport is identified. In the first two scenarios, a constant flow rate (or constant Darcy's flow rate) condition was considered, which implies a change in porosity will result in a change in pore water velocity. In the third scenario, a constant head condition (Johnson et al. 2013) is assumed, and retention of CMC-NZVI particles and its implications on porous media properties are assessed. In the third scenario, a change in porosity influences hydraulic conductivity and thus Darcy's flow rates.

\section{Theory}

\subsection{Transport modeling}

A one-dimensional (1-D) colloid transport model is developed accounting for attachment, detachment, and straining mechanism, and incorporating the change in porous media properties with respect to time and space. The dynamic mass balance of CMC-NZVI colloid is expressed below:

$$
\begin{aligned}
& \frac{\partial(\varepsilon C)}{\partial t}+\rho \frac{\partial S_{\mathrm{dep}}}{\partial t}+\rho \frac{\partial S_{\mathrm{str}}}{\partial t} \\
& =\frac{\partial}{\partial X}\left(\varepsilon D_{x} \frac{\partial C}{\partial X}\right)-\frac{\partial(U C)}{\partial X} .
\end{aligned}
$$

In equation (1), the RHS represents the particle transport due to advection and dispersion, and the 2nd and the 3rd terms in LHS represent the deposition and straining rates of NZVI particles in the aqueous phase. $C\left(\mathrm{ML}^{-3}\right)$ is the suspended particle mass concentration that varies with the length $X(\mathrm{~L})$ of column and time $t(\mathrm{~T}), U\left(\mathrm{LT}^{-1}\right)$ is Darcy's velocity, $\rho\left(\mathrm{ML}^{-3}\right)$ is the bulk density of sand-packed column and $D_{x}\left(\mathrm{~L}^{2} \mathrm{~T}^{-1}\right)$ is the hydrodynamic dispersion. $D_{x}=\alpha_{L} \times U$, where, $\alpha_{L}(\mathrm{~L})$ is dispersivity. $\varepsilon$ is the porosity of the porous media, which is varying with time and space. $S_{\text {dep }}\left(\mathrm{MM}^{-1}\right)$ is the deposited mass due to combined effect of attachment and detachment over the unit mass of sand, and $S_{\mathrm{str}}\left(\mathrm{MM}^{-1}\right)$ is the strained mass of particles per unit mass of sand. The rates of deposition and straining can be represented by the following expressions

$$
\begin{aligned}
\rho \frac{\partial S_{\mathrm{dep}}}{\partial t} & =\varepsilon k_{\mathrm{dep}} C-\rho k_{\mathrm{dep}} S_{\mathrm{dep}} \\
\rho \frac{\partial S_{\mathrm{str}}}{\partial t} & =\varepsilon k_{\mathrm{str}} \psi_{\mathrm{str}} C .
\end{aligned}
$$

Equations (2) and (3) represent retained mass of NZVI particles in the solid phase due to deposition and straining, respectively. In equation (2), $k_{\text {dep }}\left(\mathrm{T}^{-1}\right)$ and $k_{\text {det }}\left(\mathrm{T}^{-1}\right)$ are the deposition and detachment rate coefficient, respectively. In equation $(3), k_{\mathrm{str}}\left(\mathrm{T}^{-1}\right)$ is the straining rate coefficient, and $\psi_{\text {str }}$ is the dimensionless colloid straining function, which is defined by $\psi=\left(d_{c} /\left(d_{c}+x\right)\right)^{\beta}$ (Bradford et al. 2003). The parameter $\beta$ describes the spatial distribution of strained particles along the length of the column. Here, $d_{c}(\mathrm{~L})$ is the average grain diameter. The solid phase deposition and straining rate are dependent on porosity $(\varepsilon)$, and deposition rate coefficient $\left(k_{\mathrm{dep}}\right)$, which are varying with respect to space and time.

$$
\begin{aligned}
\varepsilon & =\varepsilon_{0}-\frac{\rho}{\lambda \rho_{p}} S \\
k_{\mathrm{dep}} & =\left[\frac{3(1-\varepsilon) U}{2 d_{c} \varepsilon} \alpha_{\mathrm{pc}}\right] \eta_{0} .
\end{aligned}
$$

Equations (4 and 5) are constitutive relations which account for changes in porous media properties. In this model, change in porosity due to increasing solid phase volume resulted from the retained mass of NZVI particles is considered in equation (4) (Hosseini and Tosco 2013). Here, $\varepsilon_{0}$ is the initial porosity of medium before any particle retention, $\rho_{p}\left(\mathrm{ML}^{-3}\right)$ is the density of CMC-NZVI particle, and $\lambda\left(\mathrm{LL}^{-1}\right)$ is the ratio of 


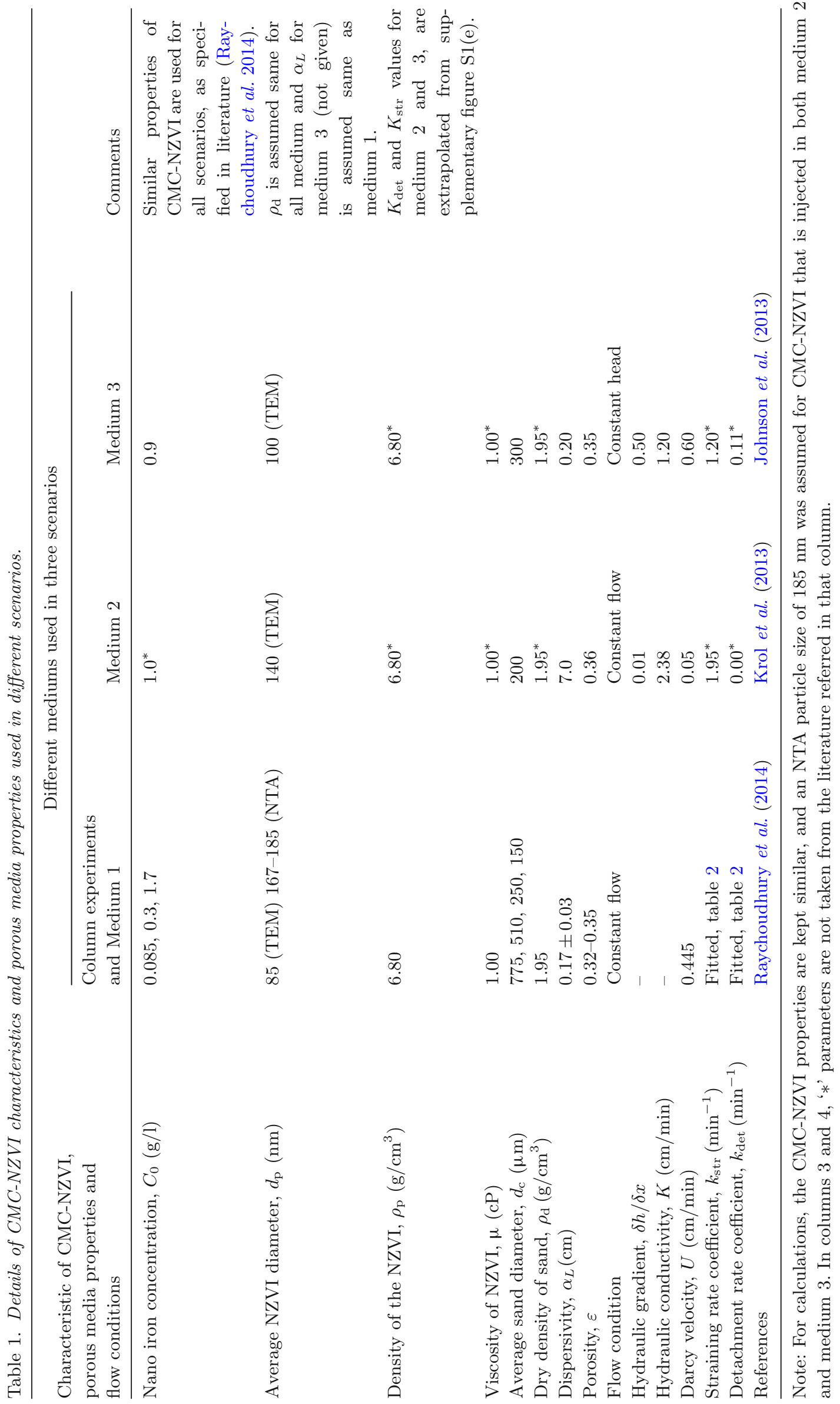


the volume of particles per unit volume of particles deposited $(0<\lambda<1)$. $S\left(\mathrm{MM}^{-1}\right)$ is the mass of retained CMC-NZVI particles over the unit mass of porous media $\left(S=S_{\text {dep }}+S_{\text {str }}\right)$. The particle deposition rate coefficient $\left(k_{\text {dep }}\right)$ described in equation (5) is related to the single-collector contact efficiency $\left(\eta_{0}\right)$, which accounts for particlesand collisions due to particle diffusion, interception, and sedimentation (Yao et al. 1971), that can be calculated using the Tufenkji and Elimelech equation (Tufenkji and Elimelech 2004). The attachment efficiency $\left(\alpha_{\mathrm{pc}}\right)$ between a particle and a collector is a fitting parameter that depends on the surface charge and solution chemistry (Tufenkji and Elimelech 2004).

For constant flow condition (scenarios 1 and 2), Darcy's velocity, $U$ will remain constant with respect to time and space. Pore-water velocity $\left(v_{\mathrm{a}}\right)$ will change inversely with porosity $\left(v_{\mathrm{a}}=U / \varepsilon\right)$. For constant head condition (scenario 3 ), the change in flow condition is accounted in equations (6) and (7). In this case, Darcy's velocity will change with hydraulic conductivity. For an incompressible fluid where the hydraulic gradient (or hydraulic head) is maintained constant, Darcy's velocity can be calculated using $U=K(d h / d l)$. Change in hydraulic conductivity due to modification in porosity is calculated using equations (6 and 7) as mentioned in the literature (Tosco and Sethi 2010), where the same expression is used to calculate the change in permeability with porosity.

$$
\begin{aligned}
K & =K_{0}\left(\frac{\varepsilon}{\varepsilon_{0}}\right)\left(\frac{a_{0}}{a}\right)^{2}, \\
a & =a_{0}+\theta a_{p} \frac{\rho}{\rho_{p}} S .
\end{aligned}
$$

In this study, the fluid properties (such as density and viscosity) are constant over space and time. Thus, a similar dependency of hydraulic conductivity on porosity can be assumed. Here, $K$ and $K_{0}$ are the hydraulic conductivity with and without particle deposition. $a\left(\mathrm{~L}^{-1}\right)$ and $a_{0}\left(\mathrm{~L}^{-1}\right)$ are the specific surface area (SSA) of the matrix after particle deposition and of clean bed system, respectively. Where $a_{p}$ is the SSA of NZVI particles and $\theta$ is the fraction of surface area of deposited particles contribute to the solid-liquid interface area $(0>\theta>1)$. The SSA of sand and particles can be calculated from their size. Sensitivity analysis for $\theta$ is carried out, and a suitable value is adopted for further analysis as described in the following sections.

\subsection{Numerical implementation}

Applying pseudo-steady state, i.e., keeping all the states and parameters constant for an infinitesimal time, finite difference methods are applied on advection and dispersion terms on equation (1). The resulting system consisting of equations (13) are solved simultaneously using Euler's explicit approach. The stability of numerical simulations on the system of differential equations (equations 1-3) is given by

$\Delta t_{1}<2 /\left(\frac{U}{\varepsilon}+\frac{2 D_{x}}{\Delta x^{2}}+k_{d e p}+k_{s t r} \psi\right)$ from equation (1)

$\Delta t_{2}<\left(\frac{2}{k_{d e p}}\right)$

from equation (2)

$\Delta t_{3}<2$

from equation (3).

The stability is assured by taking minimum time step in equation (8), i.e., $\Delta t<\min \left(\Delta t_{1}, \Delta t_{2}, \Delta t_{3}\right)$. Here, $\Delta t$ and $\Delta x$ are the time step and unit step length, respectively. The limiting value of $\Delta t$ and $\Delta x$ depends on various parameters as mentioned in equation (8). In this study, the value of $\Delta x$ is adopted as $0.01 \mathrm{~cm}$. The values of $\Delta t$ are taken as $0.01 \mathrm{sec}$ for scenarios 1 and 3 , and $0.001 \mathrm{sec}$ for scenario 2. A smaller $\Delta t$ is adopted for scenario 2 given that the porous media has very high dispersivity (table 1). Details of different scenarios are explained in section 3 .

The parameters $\left(\beta, k_{\mathrm{str}}, k_{\mathrm{det}}\right.$, and $\left.\lambda\right)$ are estimated by fitting the above-mentioned $1-\mathrm{D}$ colloid transport model to the experimental data reported in Raychoudhury et al. (2014), where all the experiments were conducted under constant flow condition (refer scenario-1 under simulation overview). Marquardt optimization technique was used for least square error minimization and parameter estimation. The problem statement for error minimization is provided in supplementary material. The linked model is presented schematically in figure 1 .

Numerical implementation is achieved using sequential solution algorithm. Based on initial and boundary conditions and assuming pseudosteady state conditions, the resulting partial differential equations (1-3) are solved using Euler's explicit scheme satisfying the time-step criteria. After estimating the deposited and strained mass, the porosities, the deposition rate coefficients, are updated for the next time step. For, constant flow condition (see scenarios 1 and 2), Darcy's velocity, $U$ will remain constant with respect to time and space. However, for the constant head condition, the updated porous media parameters, i.e., 
Optimization model

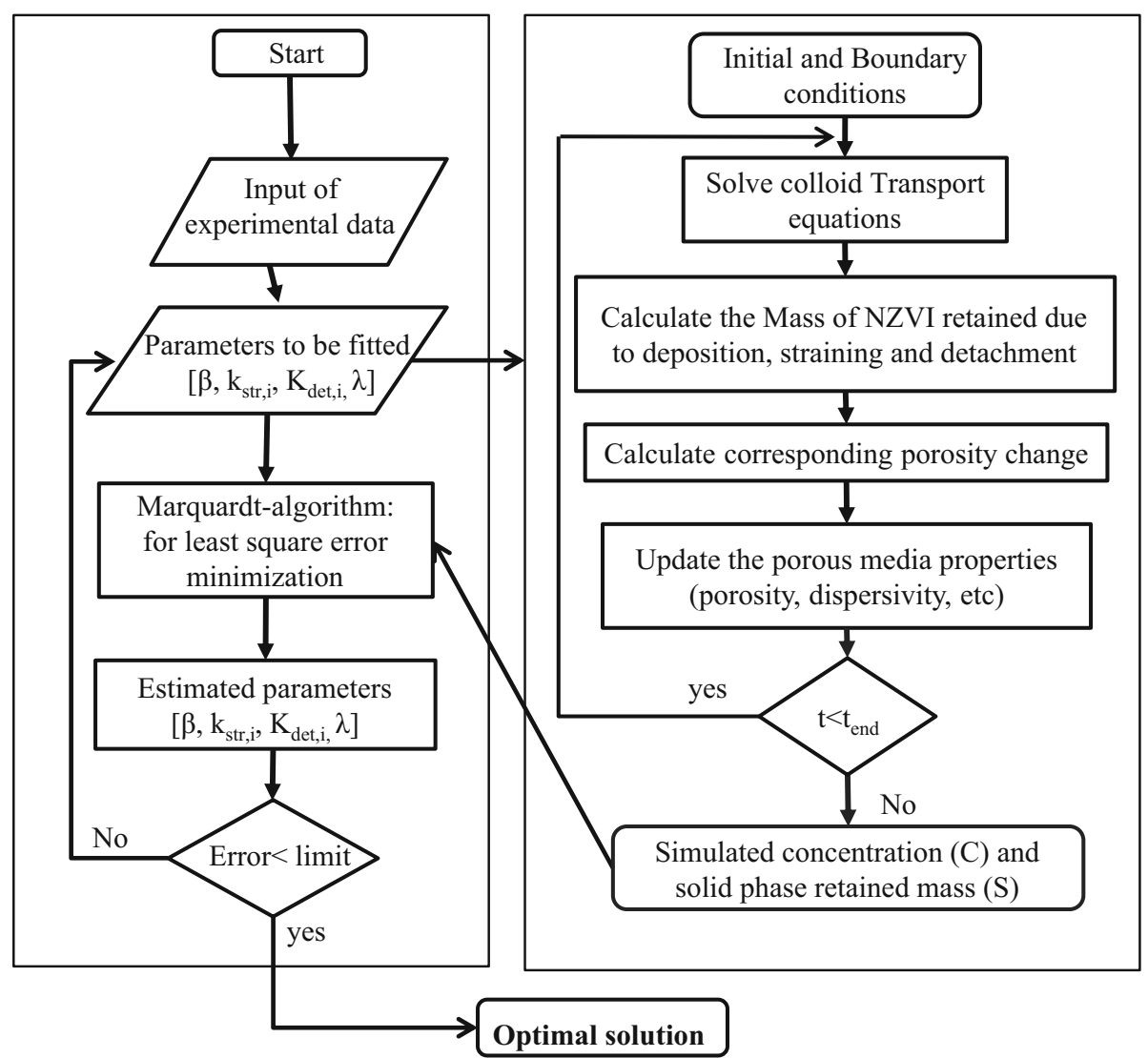

Figure 1. Schematic representation of linked optimization-colloid transport model that incorporate change in porous media properties.

porosity and the hydraulic conductivities are used for calculating Darcy's velocities in next time step. The procedure is repeated until specified integration time as recommended from the column experiments is achieved.

\section{Simulation overview}

\subsection{Scenario 1: Sand-packed column with constant flow condition}

In the first scenario, as described in Raychoudhury et al. (2014) transport of CMC-NZVI particles within various sand-packed columns having different sand-grain diameters $(150,250,510$ and $775 \mu \mathrm{m})$, is considered. The porosities of the sand columns are given as 0.32 (for $150-510 \mu \mathrm{m}$ sand) and 0.35 (for $775 \mu \mathrm{m}$ sand). The dispersivity $\left[\alpha_{L}(\mathrm{~L})\right]$ of sand-packed columns is $0.17 \pm 0.03 \mathrm{~cm}$ (see table 1). The experimental setup is presented in figure 2(a). The CMC-NZVI suspensions with concentrations of $0.085,0.35$ and $1.7 \mathrm{~g} / \mathrm{l}$, the corresponding average particle size of 167,183 , and
$185 \mathrm{~nm}$, were injected at a constant flow rate of $0.445 \mathrm{~cm} / \mathrm{min}$. Details of particle suspension, porous media properties, and flow conditions are given in table 1 . As adopted from Raychoudhury et al. (2014), the CMC-NZVI slurry was injected for 1.9 pore volumes (PVs) (except for $775 \mu \mathrm{m}$ sand-packed column, where the slurry was injected for 1.7 PVs) followed by electrolyte flushing for additional 2.8 PVs (or another $2 \mathrm{PVs}$ for $775 \mu \mathrm{m}$ sand). Same inlet flow rates were maintained into all the columns. After flow-through experiments, each column was sectioned to evaluate retained NZVI mass along the length of the column (for detailed protocol, refer Raychoudhury et al. 2014). For simulating this scenario, a constant flow boundary condition is assumed with the same rate of CMC-NZVI or electrolyte injection that is adopted in the experiment. The colloid transport model is linked with Marquardt optimization model for least square error minimization. The parameters (such as $\beta, k_{\mathrm{srt}}, k_{\mathrm{det}}, \lambda$ ) were estimated by minimizing the error between the experimental data and simulated results. 


\subsection{Scenario 2: Hypothetical 1-D porous media with constant flow condition}

In this scenario, two hypothetical porous media of $100 \mathrm{~cm}$ length are assumed; see media 1 and 2 in figure 2(b). The characteristics of one of the porous media (medium 1) are taken same as described in scenario 1, where the average sand grain of $510 \mu \mathrm{m}$ size, the porosity of 0.32 and dispersivity of $0.2 \mathrm{~cm}$ are considered. The hypothetical porous medium 2 contains sand grains of size $200 \mu \mathrm{m}$ with porosity 0.36 , and dispersivity 7.0 $\mathrm{cm}$ (table 1). The characteristic of porous medium 2 is similar to the field condition reported in the literature (Krol et al. 2013). Though the CMCNZVI characteristics are different (table 1) for the studies performed in Raychoudhury et al. (2014) (medium 1) and Krol et al. (2013) (medium 2), to compare the effect of different porous media properties on CMC-NZVI transport, characteristics of CMC-NZVI are kept constant for simulation. Inlet concentration of CMC-NZVI particles of size $185 \mathrm{~nm}$ is kept constant at $1 \mathrm{~g} / 1$ and injected for about $30 \mathrm{~min}$ (figure $2 \mathrm{~b}$ ). The initial CMCNZVI concentrations within the porous media are assumed to be zero. The constant flow condition with Darcy's velocities of $0.445 \mathrm{~cm} / \mathrm{min}$ for porous medium 1 and $0.05 \mathrm{~cm} / \mathrm{min}$ for porous medium 2 (within the range of $0.02-0.1 \mathrm{~cm} / \mathrm{min}$ that is reported in Krol et al. 2013) are assumed throughout the system.

(a)
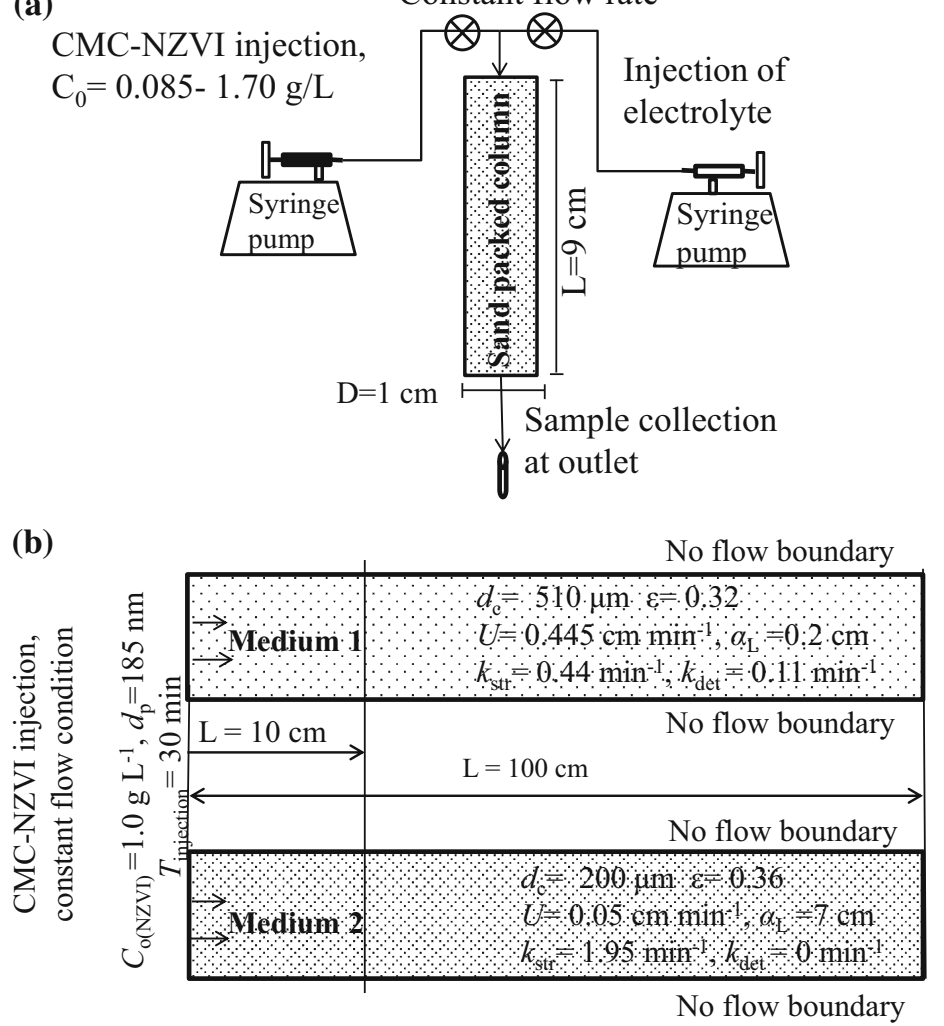

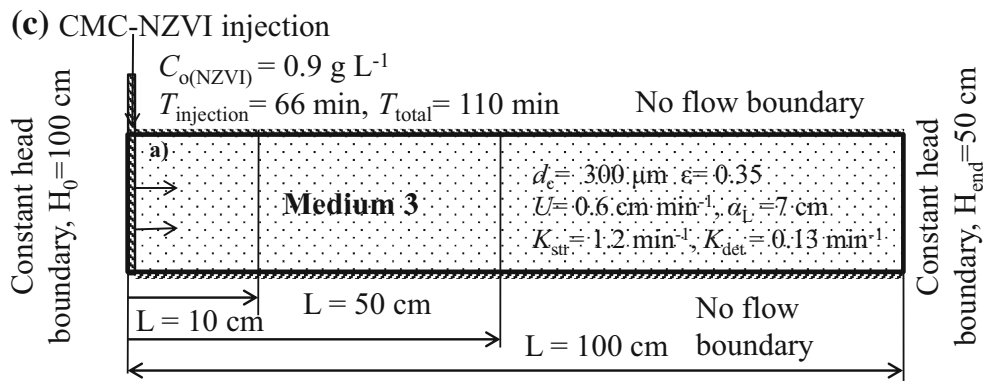

Figure 2. (a) Schematic of column transport experimental set-up adopted in Raychoudhury et al. (2014). (b) Two different hypothetical scenarios with porous medium 1 and medium 2. (c) Hypothetical study area with porous medium 3 similar to that reported in Johnson et al. (2013) having constant flow condition. 


\subsection{Scenario 3: Hypothetical 1-D aquifer layer with the constant head condition}

In this scenario, a hypothetical 1-D aquifer layer of $100 \mathrm{~cm}$ length is considered as porous medium 3; see figure 2(c) and table 1 . The hypothetical porous medium had an average grain size of $300 \mu \mathrm{m}$ and porosity of 0.35 . A constant hydraulic gradient 0.5 $\mathrm{cm} / \mathrm{cm}$ was applied across porous medium 3 with an initial hydraulic conductivity of $1.2 \mathrm{~cm} / \mathrm{min}$ and Darcy's velocity of $0.6 \mathrm{~cm} / \mathrm{min}$. The porous media properties and the flow condition of porous medium 3 are taken same as the aquifer material and the field condition reported in the literature (Johnson et al. 2013). The characteristics of injected CMCNZVI particles are considered same as described in scenarios 1 and 2. For simulating this Scenario, CMC-NZVI slurry of $0.9 \mathrm{~g} / \mathrm{l}$ concentration (similar to that used in Johnson et al. 2013) is considered to be injected for 66 mins at one end of the porous medium (one boundary), followed by groundwater flow (zero CMC-NZVI concentration) with same flow condition for another 44 mins. This time span is kept same as reported in Johnson et al. (2013). Constant head of 100 and $50 \mathrm{~cm}$ are considered at two boundaries that are $100 \mathrm{~cm}$ apart, resulted in a hydraulic gradient of $0.5 \mathrm{~cm} / \mathrm{cm}$. For simulating the concentration profile in the given system, equations (6 and 7) are incorporated along with equations (1-5). Effect of porosity on hydraulic conductivity and thus on Darcy's velocity is considered. Based on the change in hydraulic conductivity Darcy's velocity is calculated at each time interval. The parameter, $\theta$, representing the fraction of deposited particles that contributes to cover the sand-liquid interfacial is taken from Hosseini and Tosco (2013), given that the working flow rate is in the same range. Furthermore, sensitivity analysis for $\theta$ is carried out, which suggests the insignificant influence of $\theta$ on the break-through curve (BTC).

\section{Results and discussions}

\subsection{Scenario 1: Result of column transport experiment with constant flow condition}

In this scenario, the model parameters are estimated by fitting the 1-D colloid transport model (equations 1-6) to the data reported in Raychoudhury et al. (2014). In that study, the column transport experiments were conducted with four different sized sand grains (ranging from 150 to
$775 \mu \mathrm{m})$ and with three different concentrations (ranging from 0.085 to $1.7 \mathrm{~g} / \mathrm{l}$ ), as described in previous section (table 1). Estimated parameters in the previous model (Raychoudhury et al. 2014), which consider deposition, detachment, and straining mechanism, are compared with that estimated using the present model. In addition to the abovementioned mechanisms, the present model (equations 1-5) also incorporates the change in porosity due to CMC-NZVI retention and its subsequent effect on deposition rate coefficient with respect to space and time.

The BTC and deposition profiles obtained from the experiments (Raychoudhury et al. 2014) conducted with $1.7 \mathrm{~g} / \mathrm{l}$ CMC-NZVI and the corresponding simulated results are presented in figure 3(a and b) (refer supplementary figure $\mathrm{S} 1(\mathrm{a}-\mathrm{d})$ for other concentrations). The value of attachment efficiency, $\alpha_{\mathrm{pc}}$, is estimated $\left(\alpha_{\mathrm{pc}}=0.013\right)$ by fitting the simulated results with the BTC and retention profile data obtained for CMC-NZVI having lowest concentration and that is moving through the coarsest sand $(775 \mu \mathrm{m})$ matrix. In this particular case, straining will be negligible, and the change in porosity due to excess particle deposition is insignificant. This is because at a low concentration smaller sized particles are moving through comparatively bigger sized pore network, which minimizes/eliminates the effect of straining (discussed in Raychoudhury et al. 2014). Further, limited deposition is not sufficient for any observable change in porosity. Under these circumstances, $\alpha_{\mathrm{pc}}$ is fitted to the above-specified data, without considering the straining, detachment, and change in porosity. The same value of $\alpha_{\mathrm{pc}}$ is reported for the same condition in the literature (Raychoudhury et al. 2014). The deposition rate was calculated based on the same value of $\alpha_{\mathrm{pc}}$ for all the other scenarios, given the fact that $\alpha_{\mathrm{pc}}$ is dependent on solution chemistry (Tufenkji and Elimelech 2004; Raychoudhury et al. 2012), which remain constant in this set of experiments. The fitted value of parameter $\lambda$ is 1 . At the initial time, the calculated value of single collector contact efficiency $\left(\eta_{0}\right)$, and deposition rate coefficient $\left(k_{\mathrm{dep}}\right)$ match well with the values (refer table 2) reported in Raychoudhury et al. (2014). However, with the present model, the $k_{\text {dep }}$ values are changing over time and space depending on the distribution of retained CMCNZVI mass or distribution of porosity (figure $3 \mathrm{~b}$ ). The decrease in porosity with an increase in 
(a)

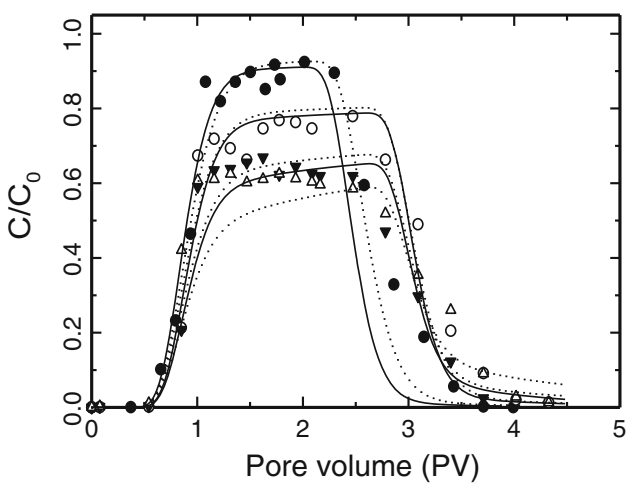

(c)

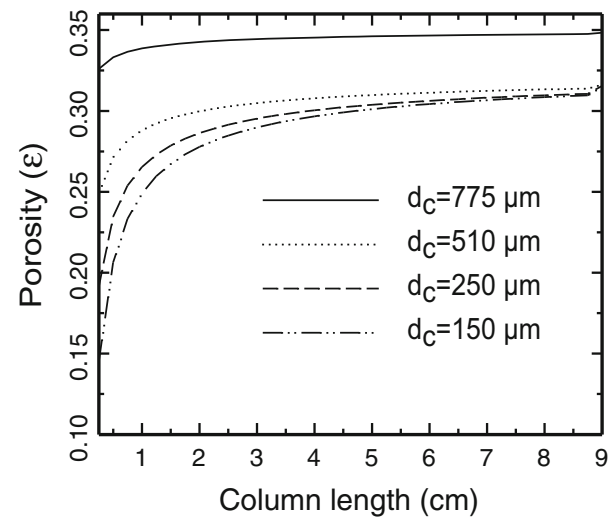

(b)

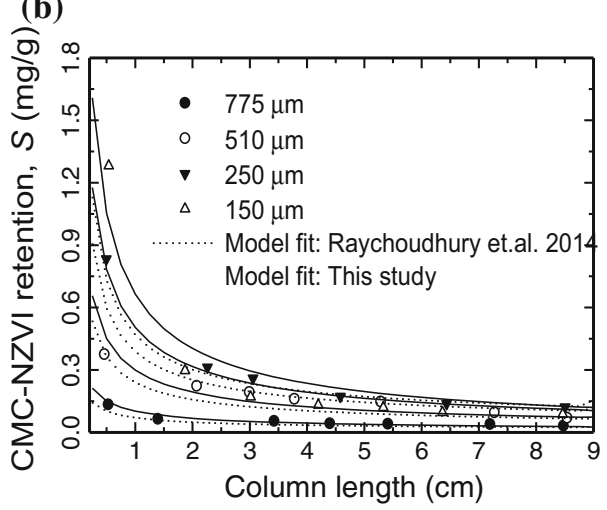

(d)

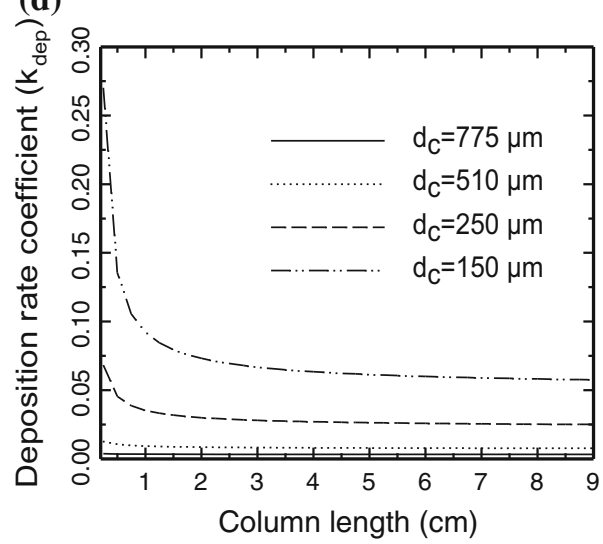

Figure 3. Experimental data and model fit for (a) breakthrough curve and (b) retention profile of CMC-NZVI. Change in (c) porosity and (d) deposition rate coefficient along the column length at the end of flushing. In this figure concentration of CMC-NZVI is $1.7 \mathrm{~g} / \mathrm{l}$. All other conditions same as shown in table 1.

retained mass can result in an increase in $\eta_{0}$ and $k_{\text {dep }}$ values (Tufenkji and Elimelech 2004). At higher CMC-NZVI inlet concentrations, in the finer sand column, the retained mass of particles due to straining is comparatively higher (figure $3 \mathrm{~b}$ ), and the subsequent decrease in porosity is more prominent (figure 3c) than those performed with coarser sands and with less concentrated CMC-NZVI. For example, our calculation suggests the porosity has decreased almost 50\%, 38\% and $25 \%$ for 150,250 , and $510 \mu \mathrm{m}$ sand respectively at the column inlet $(<0.2 \mathrm{~cm}$ from inlet), whereas for the column containing coarsest sand $(775 \mu \mathrm{m})$, insignificant $(<1 \%)$ decreases in porosity was observed even when $1.7 \mathrm{~g} / \mathrm{l}$ CMC-NZVI was injected (figure 3c). Likewise, a spatial distribution of $k_{\text {dep }}$ value is also predicted, which has an inverse trend of porosity (figure $3 \mathrm{c}$ and $\mathrm{d}$ ). At the column inlet, the value of $k_{\text {dep }}$ increases 5 times for $150 \mu \mathrm{m}, 3$ times for $250 \mu \mathrm{m}$, and 1.6 times for $510 \mu \mathrm{m}$ sized sand (figure $3 \mathrm{~d}$, table 2 ), with respect to the corresponding initial values of $k_{\mathrm{dep}}$. Some of the recent studies (Tosco and Sethi 2010; Hosseini and Tosco 2013) have suggested pore clogging phenomena due to modified-NZVI particle transport through porous media.

The model parameters for straining, i.e., $k_{\text {str }}$ and $\beta(=0.61)$ are estimated using the present model. The values of straining rate coefficients are either very close or increase insignificantly (supplementary figure S1e, table 2) compared to the value reported in Raychoudhury et al. (2014). Slight increase in straining rate coefficient, especially for finer sands, could be attributed to the fact that high particle retention near column inlet resulted in a decrease in porosity and/pore spaces, which might enhance the extent of straining. The fitted value of detachment rate coefficient is higher in this study (except the coarsest sand, table 2) compared to that reported in Raychoudhury et al. (2014). Furthermore, an increasing trend in detachment rates with a decrease in sand size (ranging from 510 to $150 \mu \mathrm{m}$ ) was observed (table 2). Similar observations were reported in Phenrat et al. (2010b). 
Table 2. Column transport experiment results and estimated parameters.

\begin{tabular}{|c|c|c|c|c|c|c|c|c|c|c|}
\hline \multirow[b]{2}{*}{$d_{\mathrm{c}}(\mu \mathrm{m})$} & \multirow[b]{2}{*}{$C_{0}(\mathrm{~g} / \mathrm{l})$} & \multirow[b]{2}{*}{$C / C_{0}$} & \multicolumn{3}{|c|}{ Raychoudhury et al. (2014) } & \multicolumn{5}{|c|}{ This study } \\
\hline & & & $\begin{array}{l}k_{\mathrm{dep}} \times 10^{-2} \\
\left(\min ^{-1}\right)\end{array}$ & $\begin{array}{c}k_{\mathrm{det}} \\
\left(\min ^{-1}\right)\end{array}$ & $\begin{array}{c}k_{\mathrm{str}} \\
\left(\min ^{-1}\right)\end{array}$ & $\begin{array}{c}k_{\mathrm{dep}} \times 10^{-2} \\
\left(\min ^{-1}\right)\end{array}$ & $\begin{array}{c}k_{\mathrm{det}} \\
\left(\min ^{-1}\right)\end{array}$ & $\begin{array}{c}k_{\mathrm{str}} \\
\left(\min ^{-1}\right)\end{array}$ & $\mathrm{r}_{\mathrm{C} / \mathrm{C}_{0}}^{2}{ }^{\mathrm{a}}$ & $\mathrm{r}_{\mathrm{S}}^{2 \mathrm{~b}}$ \\
\hline 775 & 0.085 & 0.95 & 0.34 & 0 & 0 & 0.34 & 0 & 0.00 & 0.99 & 1.00 \\
\hline 775 & 0.35 & 0.97 & 0.34 & 0.16 & 0.096 & $0.33-0.34$ & 0.16 & 0.11 & 0.94 & 0.99 \\
\hline 775 & 1.70 & 0.89 & 0.34 & 0.16 & 0.096 & $0.33-0.37$ & 0.16 & 0.11 & 0.97 & 0.99 \\
\hline 510 & 0.085 & 0.84 & 0.80 & 0.09 & 0.33 & $0.80-0.82$ & 0.11 & 0.35 & 0.98 & 0.72 \\
\hline 510 & 0.35 & 0.82 & 0.76 & 0.09 & 0.40 & $0.76-0.83$ & 0.11 & 0.44 & 0.93 & 0.96 \\
\hline 510 & 1.70 & 0.77 & 0.75 & 0.09 & 0.40 & $0.78-1.27$ & 0.11 & 0.44 & 0.95 & 0.87 \\
\hline 250 & 0.085 & 0.57 & 2.54 & 0.05 & 1.13 & $2.54-2.69$ & 0.13 & 1.54 & $0.9 \mathrm{~s} 5$ & 0.96 \\
\hline 250 & 0.35 & 0.67 & 2.38 & 0.05 & 1.00 & $2.38-2.86$ & 0.13 & 1.20 & 0.94 & 0.99 \\
\hline 250 & 1.70 & 0.60 & 2.35 & 0.05 & 1.00 & $2.35-6.82$ & 0.13 & 1.20 & 0.95 & 0.99 \\
\hline 150 & 0.085 & 0.55 & 5.81 & 0.43 & 2.15 & $5.81-6.2$ & 0.54 & 2.25 & 0.99 & 0.99 \\
\hline 150 & 0.35 & 0.62 & 5.43 & 0.43 & 1.97 & $5.43-7.1$ & 0.54 & 2.27 & 0.90 & 1.00 \\
\hline 150 & 1.70 & 0.60 & 5.40 & 0.43 & 1.97 & $5.40-27.0$ & 0.54 & 2.27 & 0.90 & 0.99 \\
\hline
\end{tabular}

Note. $^{\mathrm{a}}: \mathrm{r}_{\mathrm{C} / \mathrm{C}_{0}}^{2}=\mathrm{r}^{2}$ value for fitted model values and experimental data points for breakthrough curve. ${ }^{\mathrm{b}}: \mathrm{r}_{\mathrm{s}}^{2}=\mathrm{r}^{2}$ value for fitted model values and experimental data points for retention profile along the column length.

In our study, a decrease in porosity due to particle retention resulted in an increase in pore water velocity (figure S2). This phenomenon is more prominent in finer sand columns and negligible in coarsest sand columns. In a constant flow system, Hosseini and Tosco (2013) have demonstrated that pore clogging results in increasing pressure drop, which eventually increases particle detachment. Similar observations are made through our model parameter estimation (table 2), where more particle detachment in the finer sand was observed. It is more likely that an increase in pore water velocity resulted from a decrease in porosity, is attributed to more particle detachment, especially in finer sands.

\subsection{Scenario 2: Assessing the influence of porous media properties on $C M C-N Z V I$ transport}

In this scenario, CMC-NZVI transport behaviour in two different porous media (medium 1: figure 2a and medium 2: figure $2 \mathrm{~b}$ ), under constant flow condition, is compared. Inlet flow rates and porous media properties of media 1 and 2 are different as described in simulation overview section (figure $2 \mathrm{~b}$, table 1). For simulating scenario 2, equation (4) is considered, which incorporate change in porosity due to retention of solid phase CMC-NZVI mass, but the Darcy's flow rate remains constant. The value of $\lambda$, used to calculate the change in porosity, is assumed same $(\lambda=1)$ as determined in scenario 1. The detachment rate for medium 2 is assumed to be zero, which has a nominal flow rate. The sand grain size considered in medium 1 is larger than medium 2. A higher straining rate coefficient $\left(k_{\mathrm{str}}=1.95 \mathrm{~min}^{-1}\right)$ for medium 2 is extrapolated from supplementary figure S1(e), which indicates a linear relationship between straining rate coefficient and CMC-NZVI particle size to sand grain ratio $\left(d_{\mathrm{p}} / d_{\mathrm{c}}\right)$. This relationship was established based on column transport experiments conducted with uniform sized sand in clean laboratory system (table 1 ). Where $k_{\text {str }}$ value for medium 1 is taken as $0.44 \mathrm{~min}^{-1}$ (as fitted for $510 \mu \mathrm{m}$ sand). In both the porous media same CMC-NZVI slurry of $1 \mathrm{~g} / \mathrm{l}$ concentration is injected for $30 \mathrm{~min}$.

The aqueous phase concentrations of CMCNZVI particles are calculated at a distance of 10 $\mathrm{cm}$ from the point of injection for both media 1 and 2 . The relative concentration $\left(C / C_{0}\right)$ is presented in figure 4(a). In the case of medium 1 , it is observed that approximately $80 \%$ of injected CMCNZVI particles are transported beyond $10 \mathrm{~cm}$ of the column. Whereas, in medium 2 only around $10 \%$ of injected particles are transported. The steady state spatial profiles of relative concentrations are shown in figure 4(b). It is observed that CMS-NZVI particles transported up to a greater distance of $50 \mathrm{~cm}$ in the case of medium 1, compared to a distance of $20 \mathrm{~cm}$ for medium 2 .

An attempt is made to isolate the effects of various factors, such as straining, detachment, dispersivity, flow rate, etc., on CMC-NZVI transport. This analysis is carried out to gain insight on 

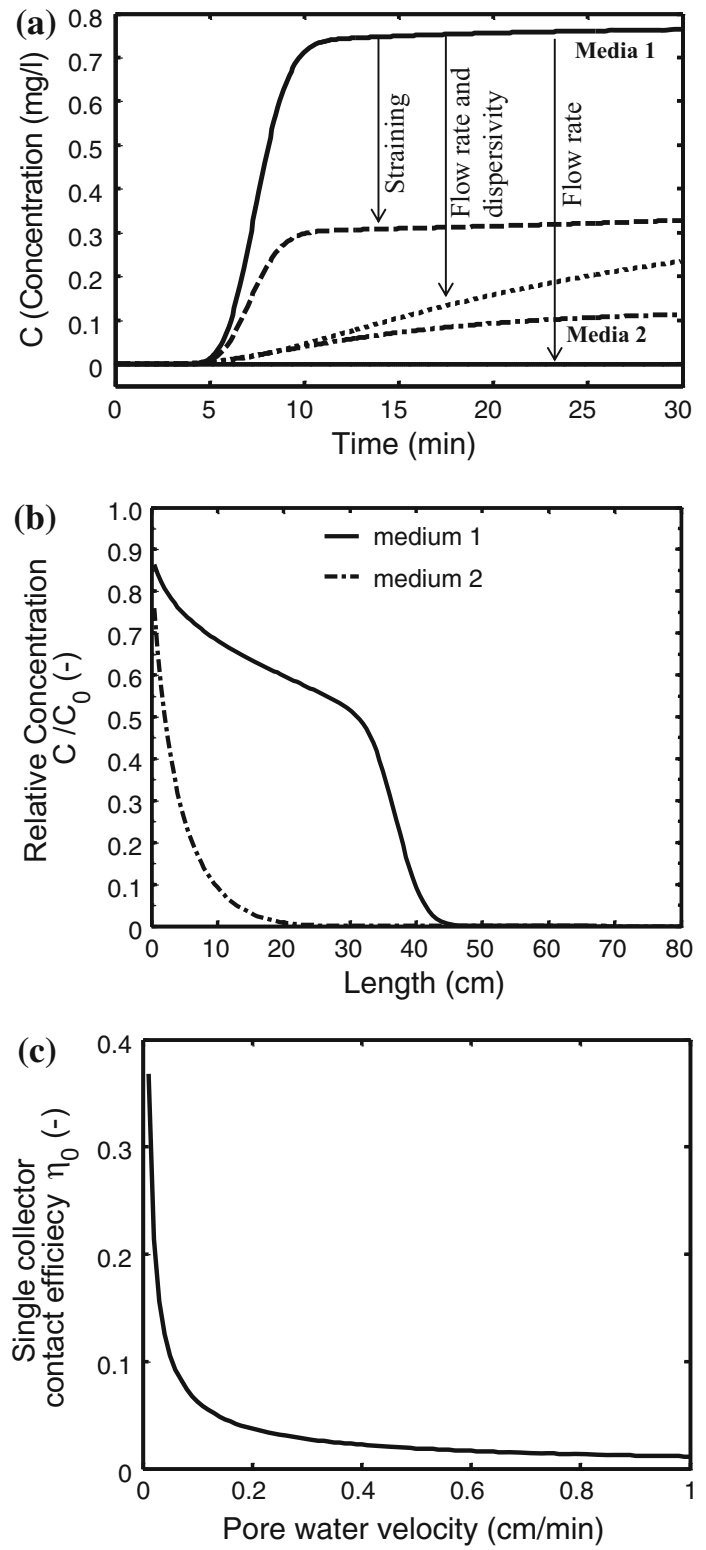

Figure 4. (a) Relative concentration of CMC-NZVI with respect to time at a distance of $10 \mathrm{~cm}$ from injection point and (b) with respect to space at the end of CMC-NZVI injection. (c) Effect of pore water velocity on single collector contact efficiency.

the influence of different transport mechanism on the retention of particles in porous media having different properties. To simulate the effect of straining, all the parameters for medium 2 are assumed same as medium 1, except the straining rate coefficient, which is 4.4 times higher for medium $2\left(k_{\mathrm{str}}=1.95 \mathrm{~min}^{-1}\right)$ compared to medium $1\left(k_{\mathrm{str}}=0.44 \mathrm{~min}^{-1}\right)$. Model calculation suggests a higher rate of straining resulted in $37 \%$ more particles retention compared to medium 1 (figure 4a). This is a significant amount of mass retained due to straining, caused by sand size. It is important to note that for medium 2, a uniformly sized sand of $200 \mu \mathrm{m}$ was considered and a corresponding $k_{\text {str }}$ is adopted. However, in the natural soil matrix, graded sand with smaller pore size is expected. Bradford et al. (2003) demonstrated that the straining of colloidal latex particle is much higher when transported through graded sand, compared to uniformly sized sand. Thus, it could be said that, that is a natural aquifer, with sand of same average diameter, the extent of straining of CMC-NZVI colloid particles will be higher based on the characteristic of the soil matrix and/or graded sand.

The significance of the difference in Darcy's velocity on retention of CMC-NZVI is compared. Our calculations suggest that most of the particles $(>99 \%)$ are retained within $5 \mathrm{~cm}$ from the point of injection when the Darcy's velocity is adjusted to $0.05 \mathrm{~cm} / \mathrm{min}$ to medium 1 . Figure $4(\mathrm{a})$ indicates that none or negligible $(<0.005 \%)$ amount of CMC-NZVI particles have reached $10 \mathrm{~cm}$ distance from the point of injection. It could be observed that 10 times reduction in Darcy's velocity, keeping dispersivity as same, resulted in $80 \%$ more particle retention (figure $4 \mathrm{a}$ in the porous media). To understand the effect of velocity on the particle deposition, the value of single collector contact efficiency was calculated as a function of pore water velocity, keeping all the other parameters constant. From figure 4(c), for the given conditions (similar to medium 1), it could be observed that the pore water velocity above $0.2 \mathrm{~cm} / \mathrm{min}$ does not influence $\eta_{0}$ value significantly. However, reduction in flow rate below $0.2 \mathrm{~cm} / \mathrm{min}$ resulted in a rapid increase in collision frequency between the particles and sand grain $\left(\eta_{0}\right)$. This is responsible for high particle deposition with a reduction in flow rate; although model calculations show, at low Darcy's flow rate, no particles are transported beyond 5 $\mathrm{cm}$ distance. However, figure 4(c) indicated that around $11 \%$ of injected particles have travelled through $10 \mathrm{~cm}$ in medium 2 . These differences are primarily governed by the difference in dispersivity. Dispersivity of medium 2 is 35 times higher than that of medium 1 , and dispersion process is also dependent on flow rate. Once the difference in flow rate and the dispersion was combined, by considering Darcy's velocity of $0.05 \mathrm{~cm} / \mathrm{min}$ with dispersivity of $7 \mathrm{~cm}$ for medium 2, keeping other parameters same as medium 1 , the model calculation suggests around 23\% CMC-NZVI particles have travelled the same distance. The effect of detachment is found to have (plot not shown) 
negligible contribution on the particle transport, especially with the flow condition adopted in this scenario.

Porous media properties and flow conditions considered for medium 2 are similar to that reported in Krol et al. (2013). Their study indicates that Darcy's velocity decreases with time when the constant head condition was adopted. Furthermore, the hydraulic head (or pressure) at the injection well increases, when the constant rate of injection was applied. The authors suggested that the decrease in viscosity due to dilution and/or by passing of $\mathrm{CMC}$ results in decreases in flow rate with time. They have also indicated that the decrease in viscosity due to change in CMC concentration is responsible for an increase in $\eta_{0}$ and $k_{\text {dep }}$. For our simulations, we have made a similar observation of an increasing trend in $\eta_{0}$ and $k_{\text {dep }}$ with time, especially near the point of injection (as discussed in the previous section). A decrease in Darcy's velocity was also observed through simulation when the constant head condition was assumed (supplementary figure S2). It is important to note that the characteristics of CMC-NZVI considered in scenario 2 (that is injected into medium 2) are very different than that reported in Krol et al. (2013). Change in viscosity with time is not likely for this particular CMC-NZVI particles (Raychoudhury et al. 2014), and thus not considered in this study. However, our model calculation suggests that due to retention of excessive CMCNZVI mass, the porosity of the media changes, which is responsible for a decrease in Darcy's velocity (when the constant head condition is used) and an increase in deposition rate (in both constant head and constant flow injection conditions).

\subsection{Scenario 3: Discussion on CMC-NZVI transport in 1-D aquifer with constant head condition}

In this scenario, one-dimensional hypothetical aquifer layer, with constant head conditions that resulted in an initial Darcy's velocity of $0.6 \mathrm{~cm} / \mathrm{min}$, is considered. The aquifer characteristics are taken same as reported in Johnson et al. (2013) (presented in figure $2 \mathrm{c}$ and in table 1 , as well as described in simulation overview). For simulations, the concentration of CMC-NZVI is taken as 0.9 $\mathrm{g} / \mathrm{l}$ with average particle size of $185 \mathrm{~nm}$ (similar to that taken in scenario 2). The CMC-NZVI slurry was injected for $66 \mathrm{~min}$ duration, which is carried out by the groundwater flow $(0.6 \mathrm{~cm} / \mathrm{min})$ for another $44 \mathrm{~min}$. In this scenario, the hydraulic head at the boundary and within the medium 3 is considered as constant over the entire period. Solid phase deposited mass (figure S3a) along the medium resulted in a reduction in porosity and hydraulic conductivity (figure S3b). To calculate the change in porosity, the same value of $\lambda(=1)$ as fitted earlier was considered. For the calculation of hydraulic conductivity, the value of fitting parameter $\theta$ (equations 6 and 7 ) is taken as 0.0004 in the range given in Tosco and Sethi (2010). Furthermore, the sensitivity of $\theta$ on hydraulic conductivity and on CMC-NZVI concentration profile is evaluated, which shows that value of $\theta$, within that reported range of 0 to 1 (Tosco and Sethi 2010), has insignificant influence on hydraulic conductivity and on CMC-NZVI transport. The detachment rate of CMC-NZVI was taken as $0.11 \mathrm{~min}^{-1}$ (as specified in table 1), within the range identified in the column transport study (table 2).

Transport behaviour of CMC-NZVI and the effect of CMC-NZVI particle retention on porous media properties, Darcy's velocity, and on further particle deposition is evaluated from the model calculation (figure 5, supplementary figure S3). The concentration of CMC-NZVI along time, at 25, 50, and $100 \mathrm{~cm}$ distance from the point of injection are calculated and presented in figure $5(\mathrm{a}-\mathrm{c})$. It could be observed that at a distance of 25,50 , and $100 \mathrm{~cm}$, peak concentration reaches up to approximately 265, 200 and $125 \mathrm{mg} / \mathrm{l}$ (with considering detachment) after 25, 55 and $85 \mathrm{~min}$ from the start of injection. A detachment of deposited CMCNZVI particles is responsible for a tailing effect (supplementary figure figure S3c), even though in scenario 2, the detachment is identified to have an insignificant effect on CMC-NZVI transport, compared to the effect of straining and flow rate. However, with longer time and at a greater distance, when the concentration of CMC-NZVI reduces, the effect of detachment has a greater role. For example, at a distance of $25 \mathrm{~cm}$, the increase in peak particle concentration due to particle detachment $\left(k_{\operatorname{det}}=0.11\right)$ is $13 \%$; however, at a distance of 50 and $100 \mathrm{~cm}$, the increase in peak concentration due to same rate of detachment are around $45 \%$ (110 $200 \mathrm{mg} / \mathrm{l})$ and $68 \%(40-125 \mathrm{mg} / \mathrm{l})$ respectively, (figure $5 \mathrm{a}-\mathrm{c}$ ).

Due to extensive particle retention, near the point of injection, the hydraulic conductivity of the porous medium has also reduced significantly (supplementary figure S3c). For example, at a distance of $0.5 \mathrm{~cm}$, the hydraulic conductivity decreases 
(a)

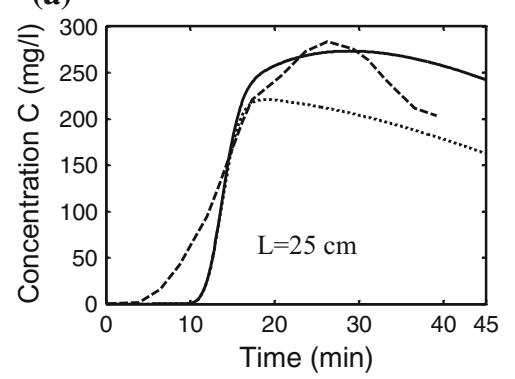

(d)

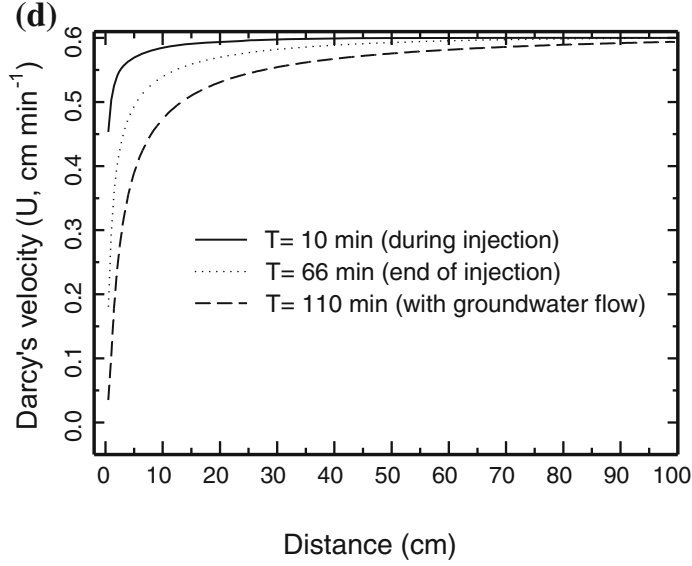

(b)

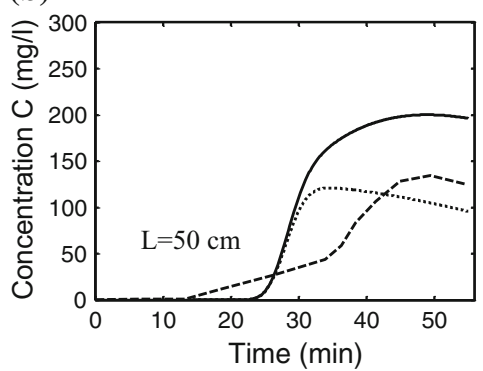

(c)

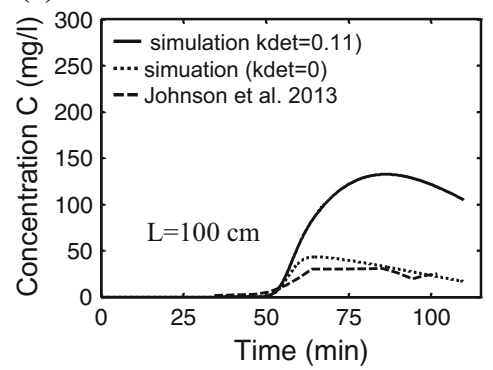

(e)

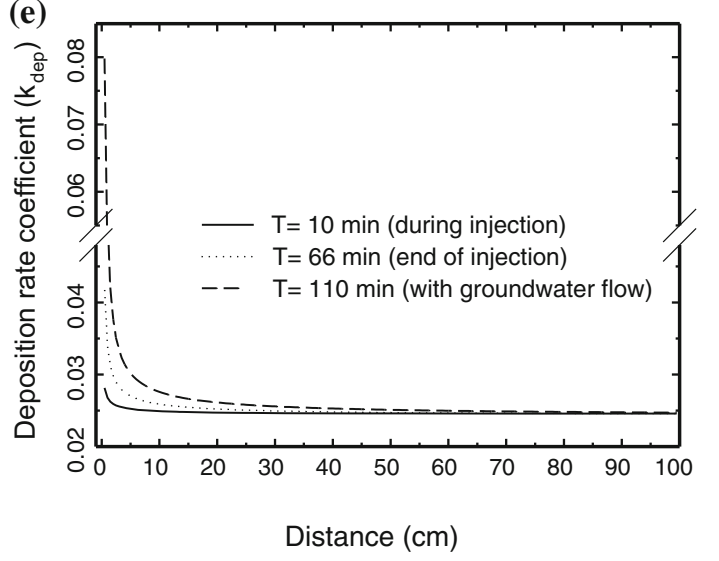

Figure 5. CMC-NZVI concentration with time at $(\mathbf{a})$ distance, $\mathrm{L}=10 \mathrm{~cm},(\mathbf{b})$ at a distance, $\mathrm{L}=50$, and $(\mathbf{c})$ distance, $\mathrm{L}=100$ $\mathrm{cm}$ from the injection well. (d) Change in Darcy's velocity and (e) deposition rate coefficient along space.

almost 12 times after 110 min compared to that of the initial value. With the decrease in hydraulic conductivity, Darcy's velocity also decreases proportionally. At the same distance of $0.5 \mathrm{~cm}$ and at the end of $110 \mathrm{~min}$, Darcy's velocity reduces from 0.6 to $0.05 \mathrm{~cm} / \mathrm{min}$ (12 times, figure $5 \mathrm{~d}$ ). Retention of CMC-NZVI and subsequent change in porosity and Darcy's velocity resulted in an increase in deposition rate coefficient as shown in figure 5(e).

As specified above, the aquifer characteristic and flow conditions are kept similar to the field condition reported in Johnson et al. (2013). In their study, $0.9 \mathrm{~g} / \mathrm{l}$ of NZVI, in the presence of CMC, was injected, and at a distance of 25, 50 and 100 $\mathrm{cm}$, they have identified a maximum iron concentration of approximately 275, 140 and $25 \mathrm{mg} / \mathrm{l}$ after 30,45 and $90 \mathrm{~min}$, respectively (figure $5 \mathrm{a}-\mathrm{c}$ derived from Johnson et al. 2013). The trend of the measured concentration profile of total iron with respect to time matches well with our model calculation including detachment at a shorter distance $(\mathrm{L}=25 \mathrm{~cm})$. However at a further distance $(\mathrm{L}=50-100 \mathrm{~cm})$, reported concentration profiles have a better match with the model calculation that does not incorporate detachment of deposited CMC-NZVI (figure 5). In this field experiment, firstly, the occurrence of chemical transformation of iron and formation of $\mathrm{H}_{2}$ gas is reported, which is likely to increase the deposition of NZVI (Johnson et al. 2013). Johnson et al. (2013) have demonstrated that the proportion of iron oxide increases with distance in the field condition. Thus, more particle retention at a greater distance compared to the model calculation (incorporating detachment) could be explained by the fact that the rate of deposition increases with distance due to chemical transformation of NZVI. Secondly in the field scenario, the groundwater chemistry, soil composition in the natural system (i.e., presence of natural organic matter or mineral composition) as well as the types of CMC-NZVI applied are different. Thus, the difference in extent and rate of particle retention between model calculation and data is expected. However, from the data and model comparison, it can be stated that straining, deposition, and alteration of porous media properties due to retention of nano-particles has an important role on CMC-NZVI transport, which can be investigated further in field studies.

There are few differences between the 1-D model prediction and the field condition consisting of three-dimensional (3-D) porous matrices. For example, (i) it is likely that the effect of straining is overestimated for field scenario ( 3 -D porous 
matrix) considering the fitted value of straining rate for the 1-D model. However, in the natural system, graded sand is likely to be present, which may have a higher straining rate compared to the fitted value (Bradford et al. 2003). (ii) The transport of NZVI is also controlled by density gradient (Phenrat et al. 2010) in a 2-D/3-D system. Thus, in addition to horizontal (along with the groundwater flow) transport of CMC-NZVI, a vertical movement and deposition of CMC-NZVI particles are also expected that was not considered in the model. (iii) Change in porosity and intense particle deposition in one direction can result in heterogeneity of the porous media in local scale. By-passing of the flow and particle deposition in other dimension is expected in the local scale. However, in global scale and for the larger duration, the porous media property can be averaged and thus, the rate of reduction in porosity, hydraulic conductivity and increased deposition might occur at a slower rate and reduced pace than that estimated in this study.

\section{Conclusions}

Straining of surface modified NZVI particles is an important phenomenon, resulting in the retention of a significant amount of particles near injection point when introduced at high concentration. The flow rate is also a governing factor influencing particle deposition. At low flow rate (i.e., $<0.2 \mathrm{~cm} / \mathrm{min}$ ), the possibility of collision between the sand grain and colloid particles increases significantly, resulting in high particle deposition. Our model calculation shows that extensive amount of CMC-NZVI particle retention can alter porous media properties in local scale. Overall a rapid decrease in porosity was observed as a result of high particle retention, especially near the injection points resulting in a spatial distribution of deposition rate coefficient. In the case of constant head condition, spatial distribution in Darcy's velocity is likely, resulted from variation in porosity and hydraulic conductivity. Comparison of simulated result with data reported from field studies suggests straining is likely to happen in real field condition, which can influence porous media properties and increase further nano-particles deposition.

\section{Acknowledgements}

The research work is mainly based on modeling of nano-particle transport, and the model is compared with data published in the literature. The major part of the work is conducted in BITS-Pilani, Hyderabad campus and IIT Patna. We would like to thank Prof. Ghoshal's Research Lab at McGill University, Canada, where previous study by the author was performed.

\section{References}

Bennett P, He F, Zhao D, Aiken B and Feldman L 2010 In situ testing of metallic iron nanoparticle mobility and reactivity in a shallow granular aquifer; J. Cont. Hydrol. 116(1-4) 35-46.

Bergendahl J and Grasso D 2000 Prediction of colloid detachment in a model porous media: Hydrodynamics; Chem. Eng. Sci. 55 1523-1532.

Bradford S A, Simunek J, Bettahar M, Van Genuchten M T and Yates S R 2003 Modeling colloid attachment, straining, and exclusion in saturated porous media; Environ. Sci. Technol. 37 2242-2250.

Cirtiu C M, Raychoudhury T, Ghoshal S and Moores A 2011 Systematic comparison of the size, surface characteristics and colloidal stability of zero valent iron nanoparticles pre- and post-grafted with common polymers; Colloid Surf. A 390(1-3) 95-104.

Fatisson J, Ghoshal S and Tufenkji N 2010 Deposition of carboxymethylcellulose-coated zero-valent iron nanoparticles onto silica: Roles of solution chemistry and organic molecules; Langmuir 26(15) 12,832-12,840.

He F, Zhang M, Qian T and Zhao D 2009 Transport of carboxymethyl cellulose stabilized iron nanoparticles in porous media: Column experiments and modeling; J. Colloid Interf. Sci. 334(1) 96-102.

He F, Zhao D Y and Paul C 2010 Field assessment of carboxymethyl cellulose stabilized iron nanoparticles for in situ destruction of chlorinated solvents in source zones; Water Res. 44(7) 2360-2370.

Hong Y, Honda R J, Myung N V and Walker S L 2009 Transport of iron-based nanoparticles: Role of magnetic properties; Environ. Sci. Technol. 43 8834-8839.

Hosseini S M and Tosco T 2013 Transport and retention of high concentrated nano-Fe/Cu particles through highly flow-rated packed sand column; Water Res. 47(1) 326338.

Johnson R L, Nurmi J T, Johnson G S O, Fan D M, Johnson R L O, Shi Z Q, Salter-Blanc A J, Tratnyek P G and Lowry G V 2013 Field-scale transport and transformation of carboxymethylcellulose-stabilized nano zero-valent iron; Environ. Sci. Technol. 47(3) 1573-1580.

Kanel S, Goswami R R, Clement T P, Barnett M O and Zhao D 2008 Two dimensional transport characteristics of surface stabilized zero-valent iron nanoparticles in porous media; Environ. Sci. Technol. 42(3) 896-900.

Kim H J, Phenrat T, Tilton R D and Lowry G V 2012 Effect of kaolinite, silica fines and $\mathrm{pH}$ on transport of polymermodified zero valent iron nano-particles in heterogeneous porous media; J. Colloid Interf. Sci. 370 (1) 1-10.

Krol M M, Oleniuk A J, Kocur C M, Sleep B E, Bennett P, Xiong Z and O'Carroll D M 2013 A field-validated model for in situ transport of polymer-stabilized NZVI 
and implications for subsurface injection; Environ. Sci. Technol. 47(13) 7332-7340.

Laumann S, Miciä V and Hofmann T 2014 Mobility enhancement of nanoscale zero-valent iron in carbonate porous media through co-injection of polyelectrolytes; Water Res. 50 70-79.

O'Carroll D, Sleep B, Krol M, Boparai H and Kocur C 2012 Nanoscale zero valent iron and bimetallic particles for contaminated site remediation; Adv. Water Res. 51 104-122.

Petosa A R, Jaisi D P, Quevedo I R, Elimelech M and Tufenkji N 2010 Aggregation and deposition of engineered nanomaterials in aquatic environments: Role of physicochemical interactions; Environ. Sci. Technol. 44(17) 6532-6549.

Phenrat T, Cihan A, Kim H J, Mital M, Illangasekare Tand Lowry G V 2010a Transport and deposition of polymer-modified $\mathrm{FeO}$ nanoparticles in 2-D heterogeneous porous media: Effects of particle concentration, FeO content, and coatings; Environ. Sci. Technol. 44(23) 90869093.

Phenrat T, Kim H J, Fagerlund F, Illangasekar T and Lowry G V 2010b Empirical correlations to estimate agglomerate size and deposition during injection of a polyelectrolytemodified $\mathrm{FeO}$ nanoparticle at high particle concentration in saturated sand; J. Cont. Hydrol. 118(3-4) $152-164$

Phenrat T, Kim H J, Fagerlund F, Illangasekare T, Tilton R D and Lowry G V 2009 Particle size distribution, concentration, and magnetic attraction affect transport of polymer-modified $\mathrm{FeO}$ nanoparticles in sand columns; Environ. Sci. Technol. 43(13) 5079-5085.

Raychoudhury T, Naja G and Ghoshal S 2010 Assessment of transport of two polyelectrolyte-stabilized zero-valent iron nanoparticles in porous media; J. Cont. Hydrol. 118 143-151.

Raychoudhury T and Scheytt T 2013 Potential of zero valent iron nanoparticles for remediation of environmental organic contaminants in water: A review; Water Sci. Technol. 68(7) 1425-1439.

Raychoudhury T, Tufenkji N and Ghoshal S 2012 Aggregation and deposition kinetics of carboxymethyl cellulosemodified zero-valent iron nanoparticles in porous media; Water Res. 46(6) 1735-1744.

Raychoudhury T, Tufenkji N and Ghoshal S 2014 Significance of straining during polyelectrolyte modified iron nanoparticle transport in porous media; Water Res. 50 $80-89$.

Saleh N, Kim H J, Phenrat T, Matyjaszewski K, Tilton R D and Lowry G V 2008 Ionic strength and composition affect the mobility of surface-modified $\mathrm{FeO}$ nanoparticles in water-saturated sand columns; Environ. Sci. Technol. 42(9) 3349-3355.

Saleh N, Sirk K, Liu Y, Phenrat T, Dufour B, Matyjaszewski K, Tilton R D and Lowry G V 2007 Surface modifications enhance nanoiron transport and NAPL targeting in saturated porous media; Environ. Eng. Sci. 24(1) 45-57.

Su C M, Puls R W, Krug T A, Watling M T, O'Hara S K, Quinn J W and Ruiz N E 2013 Travel distance and transformation of injected emulsified zerovalent iron nanoparticles in the subsurface during two and half years; Water Res. 47(12) 4095-4106.

Tosco T and Sethi R 2010 Transport of non-Newtonian suspensions of highly concentrated micro- and nanoscale iron particles in porous media: A modeling approach; Environ. Sci. Technol. 44 9062-9068.

Tratnyek P G and Johnson R L 2006 Nanotechnologies for environmental cleanup; Nano Today 1 44-48.

Tufenkji N and Elimelech M 2004 Correlation equation for predicting single-collector efficiency in physicochemical filtration in saturated porous media; Environ. Sci. Technol. 38 529-536.

Yao K M, Habibian M T and O'Melia C R 1971 Water and waste water filtration: Concepts and applications; Environ. Sci. Technol. 5(11) 1105-1112. 